\title{
Novel phenoxazine-benzonitrile and phenothiazine-benzonitrile donor-acceptor molecules with thermally activated delayed fluorescence (TADF)
}

\author{
Faiza Baraket $^{\mathrm{a}, \mathrm{b}}$, Bruno Pedras ${ }^{\mathrm{a},{ }^{*}, \text { Erica Torres }}{ }^{\mathrm{a}, \mathrm{c}, 1}$, Maria João Brites ${ }^{\mathrm{c}}$, Mohamed Dammak ${ }^{\mathrm{b}}$, \\ Mario N. Berberan-Santos ${ }^{\text {a }}$ \\ a CQFM-IN and IBB-Institute for Bioengineering and Biosciences, Instituto Superior Tecnico, Universidade de Lisboa, 1049-001, Lisboa, Portugal \\ ${ }^{\mathrm{b}}$ Laboratory of Inorganic Chemistry LR17ESO7, Faculty of Sciences of Sfax, University of Sfax, 3000, Sfax, Tunisia \\ ${ }^{\mathrm{c}}$ Laboratorio Nacional de Energia e Geologia, I. P., UER-Unidade de Energias Renovaveis e Integração de Sistemas de Energia, Edifício G, 1 Andar, Estrada do Paço do \\ Lumiar, 22, 1649-038, Lisboa, Portugal
}

\section{A R T I C L E I N F O}

\section{Keywords:}

TADF

OLED

Fluorescence

Phenoxazine

Phenothiazine

\begin{abstract}
A B S T R A C T
Four novel TADF emitters, containing phenothiazine and phenoxazine as electron-donors and benzonitrile derivatives as electron-acceptors were synthesized and fully characterized. Their photophysical (absorption and emission spectra, molar extinction coefficients, fluorescence quantum yields and lifetimes) and electrochemical properties (HOMO and LUMO energy levels) were measured, and drop-cast solid films of the four compounds were obtained to perform TADF studies. The obtained values for $\Delta \mathrm{E}_{\mathrm{ST}}$ indicate that these compounds are candidates for OLED applications.
\end{abstract}

\section{Introduction}

Luminescent materials for organic light-emitting diodes (OLEDs) have been an important subject of both academic and industrial research in recent years. Organic fluorescence emitters with Thermally Activated Delayed Fluorescence (TADF) have attracted much attention in that these materials use thermally activated up-conversion of triplet $\left(\mathrm{T}_{1}\right)$ into singlet $\left(S_{1}\right)$ state, giving stable fluorescence with very high fluorescence yields [1-3]. These TADF materials, used in third generation Organic Light Emitting Diodes (OLEDs) by charge-transfer (CT) excited state [4, 5], require a sufficiently small energy gap between the triplet and singlet $\left(\Delta \mathrm{E}_{\mathrm{ST}}\right)$ to enable up-conversion of the triplet excitons to singlet excitons and realize $100 \%$ internal quantum efficiency $[6,7]$ of the exciton formation generated by electric excitation at $S_{1}$. Thus, enormous efforts have been endeavored to minimize $\Delta \mathrm{E}_{\mathrm{ST}}$ and simultaneously enhancing the reverse intersystem crossing (RISC) by molecular structure design, leading to the maximization of TADF. Nowadays, particularly designed organic materials with donor-acceptor structures are used to minimize the HOMO-LUMO overlap.

Versatile donor-acceptor (DA) molecular systems with TADF have been developed, including acridine [8], carbazole [9], phenoxazine [10-12], phenothiazine and diphenylamine as donor groups and triazine [13], cyanobenzene, benzophenone and diphenyl sulfone derivatives [14] as acceptor groups. High external quantum efficiencies (EQE) up to $20 \%$ have been reported for OLEDs using TADF materials as emitters, which come close to the best performance of OLEDs based on organic phosphorescent materials reported.

Phenoxazine and phenothiazine are potentially good electron donors and hence have been used extensively as fluorescent emitters in first generation OLEDs [15-18], dye sensitized solar cells [19] and laser dyes [20]. However, because these materials are conventional fluorescent emitters rather than TADF ones, the EQEs obtained for their devices are less than those of phosphorescent OLEDs. In these fluorescent compounds, the large overlap between HOMO and LUMO is a major factor preventing the existence of TADF. In DA systems the Highest Occupied Molecular Orbital (HOMO) is localized in the electron donor moiety and the Lowest Unoccupied Molecular Orbital (LUMO) in the electron acceptor moiety.

Based on the aforementioned, in this work we report the design, synthesis and characterization of four novel donor-acceptor TADF

\footnotetext{
* Corresponding author.

E-mail address: bruno.pedras@tecnico.ulisboa.pt (B. Pedras). Lisboa, Portugal.

${ }^{1}$ Present address: Centro de Química Estrutural and Institute of Nanosciences and Nanotechnology, Instituto Superior Tecnico, Universidade de Lisboa, 1049-001 